\title{
Effect of periodic gas-puffs on drift-tearing modes in ADITYA/ADITYA-U Tokamak
}

\section{discharges}

Harshita Raj ${ }^{1 *, 2}$, Tanmay Macwan ${ }^{1,2}$, Kaushalendra Singh ${ }^{1,2}$, Suman Dolui ${ }^{1,2}$, Joydeep Ghosh $^{1,2}$, Nirmal K Bisai ${ }^{1,2}$, K. A. Jadeja ${ }^{2}$, K. M. Patel ${ }^{2}$, N. C. Patel ${ }^{2}$, R. L. Tanna ${ }^{2}$, D. Raju ${ }^{1,2}$, S. K. Jha ${ }^{2}$, P. K. Chattopadhyay ${ }^{1,2}$, Abhijit Sen ${ }^{1,2}$, Y, C, Saxena ${ }^{1,2}$, R. Pal ${ }^{3}$, and ADITYA-U Team $^{2}$

${ }^{1}$ HBNI University, Mumbai, India

${ }^{2}$ Institute for Plasma Research, Bhat, Gandhinagar 382428, India

${ }^{3}$ Saha Institute of Nuclear Physics, Kolkata, India

\section{$\underline{\text { Abstract }}$}

The effect of a periodic train of short gas-puff pulses on the rotation frequency and amplitude of drift-tearing modes has been studied in ADITYA/ADITYA-U tokamak. The short gas puffs, injecting approximately $\sim 10^{17}-10^{18}$ molecules of fuel gas (hydrogen) at one toroidal location, are found to concomitantly decrease the drift-tearing mode rotation frequency and the mode amplitude during the period of injection and then recover back to its initial values when the gas pulse is over. This leads to a periodic modulation of the rotation frequency and amplitude of the drift-tearing modes that is correlated with the periodicity of the gas pulse injection. The underlying mechanism for this change in the mode characteristic appears to be related to gas puff induced change in the radial profile of the plasma pressure in the edge region that brings about a reduction in the diamagnetic drift frequency. Detailed experimental measurements and BOUT++ code simulations support such a reduction in diamagnetic drift frequency. Our results reveal a close interaction between the edge dynamics and core MHD phenomena in a tokamak that could help us better understand the rotation dynamics and amplitude pulsations of magnetic islands.

Current Affiliation:

Ecole Polytechnique Fédérale de Lausanne (EPFL), Swiss Plasma Center (SPC), Lausanne, Switzerland 


\section{Introduction}

Controlling the growth of tearing modes, one of the most common macro-instabilities present in a tokamak, still remains a prime objective and a challenging task for tokamak plasmas [1]. The uncontrolled growth of tearing modes, relatively slowly growing macro-instabilities, and subsequent formation of helical structures known as magnetic islands through the process of magnetic reconnection often leads to a sudden collapse of entire plasma energy [1]. Tearing modes are known to be driven by the free energy associated with the current density profile sustaining the equilibrium magnetic field [2]. Magnetic islands are radially localized structures centered on the rational flux surfaces, which satisfy $k \cdot \boldsymbol{B}=0$, where $k$ is the wavevector of perturbation and $\boldsymbol{B}$ is the equilibrium magnetic field. Large magnetic islands increase the radial plasma transport far above the collision-driven transport levels. Very large islands or simultaneous presence of more than one island can even lead to a very rapid loss of plasma confinement leading to a sudden termination of plasma also known as major disruption. The dynamics of magnetic islands in a tokamak is influenced by many factors such as the free energy sources, thermal transport [1], ion-sound wave [3], diamagnetic effects [4,5], magnetic curvature [6,7], external perturbations [8], polarisation current [9], etc.

The classical theory of resistive tearing modes (carried out in the single fluid description) leads to a purely growing mode with a growth rate that scales as a fractional power of the resistivity. However, in a realistic experimental situation the diamagnetic effects play an important role and a proper description of the tearing mode is provided by a two fluid model. In such a case the tearing mode acquires a real frequency which makes the mode rotate with the diamagnetic frequency and is called the drift tearing mode [10,11]. Furthermore, the diamagnetic drift frequency also leads to a decrease in the growth rate of the tearing mode. The relevant excitation threshold of the drift-tearing mode is the same as that of the classical resistive tearing mode, as long as the relevant electron equation of state is adiabatic. In general, the rotation frequency of islands, which is given by a combination of the plasma rotation frequency and the phase velocity of the island in the plasma frame, plays an important role in the growth, decay, and saturation of magnetic islands [12]. The rotation of magnetic islands around the tokamak in both poloidal and toroidal directions in the kilohertz $(\mathrm{kHz})$ range of frequencies is widely observed in tokamak discharges $[13,14]$. While rotating, the island interacts with the background plasma to produce rotating perturbations in the plasma pressure and velocity profiles, which in turn affect the island width [15]. Furthermore, interaction with the resistive wall may also halt the rotation of magnetic islands, leading to a slowdown of plasma rotation 
and causing a degradation of the confinement $[16,17]$. Controlling the rotation of the magnetic islands is attempted in order to produce damping forces that induce a reduction in the island size[14,16]. The external control system alters the island motion, which then, in turn, changes the plasma behaviour. The interaction of magnetic islands with the background plasma, particularly with the ion fluid velocity, is crucial to understanding and improving rotation control techniques [15].

An externally applied non-axisymmetric magnetic field is one of the simplest and most direct approaches towards control of MHD instabilities since it can interact directly with the nonaxisymmetric magnetic field of an instability [8,18]. Many tokamaks and reversed-field pinch devices now have single or multiple rows of non-axisymmetric coils/ resonant magnetic perturbation coils (RMP), external or internal to the vacuum vessel, that provide the capability to apply magnetic perturbations of different toroidal and poloidal mode numbers $[19,20]$. However, the effectiveness of RMP heavily depends on the initial state of the tearing mode, such as mode frequency and island width. Furthermore, a strong RMP can even stimulate a tearing mode even if the initial plasma is tearing stable and in some cases, it can lead to mode locking too.

There exists an abundance of experimental evidence of short gas puff (GP) pulses significantly influencing the scrape-off-layer (SOL) and edge plasma characteristics in tokamak discharges. Gas puffing experiments on the PBX-M [21], ADITYA [22], NSTX [23], and T-10 [24] have reported significant modifications in the SOL/edge plasma parameters and the SOL/edge turbulence due to the application of fuel gas-puff pulses. Apart from the modifications in SOL/edge plasma parameters and turbulence, the presence of neutrals in the edge plasma are also known to influence the global confinement [25] and play a major role in the transition from low $(\mathrm{L})$ to high $(\mathrm{H})$ confinement mode which are critical to the performance of tokamak based fusion reactors. The poloidally localized fueling is also known to influence the plasma rotation [26,27]. A flattening of the edge floating potential profile along with a reduction in edge floating potential fluctuations in the SOL/edge plasma region of ADITYA tokamak has been observed with the application of gas-puff pulse [28]. A decrease in edge density gradient and an increase in global confinement time have also been reported in these experiments. Furthermore, these gas puffs are also known to reduce the edge poloidal flow speed and cause a reversal in the toroidal flow speed due to the localized edge particle source $[22,29]$. Similar results have also been reported from STOR-M tokamak [30], where a two-fold increase in the line averaged density, decrease in electron temperature along with a reduction in the floating 
potential fluctuations have been observed by injecting gas in short pulses. A decrease in the loop voltage has also been observed, indicating an increase in the core electron temperature and global confinement in STOR-M [30]. In addition to the modifications in the characteristics of electrostatic fluctuations with the application of short gas-puff pulses, the Edge localized Modes (ELMs) are also reported to be influenced by pulsed supersonic molecular beam injection (SMBI) in K-STAR and HL-2A tokamak [31]. Bifurcation of tearing modes due to SMBI has been observed in J-TEXT tokamak [32].

In this paper, we report the influence of short gas-puff pulses on the amplitude and the rotation frequency of drift-tearing magnetic islands. The time-varying poloidal magnetic field fluctuations associated with the rotating magnetic islands are measured using two sets of Mirnov coils, with each set having 16 poloidal coils and placed at two different toroidal locations. It has been observed that the amplitude and rotation frequency of pre-existing $\mathrm{m} / \mathrm{n}=$ $2 / 1$ islands reduce significantly with the application of fuel (hydrogen) gas-puff pulses (neutral injection) of suitable magnitude in the SOL/edge region of ADITYA and ADITYA-U tokamaks. The decrement in the rotation frequency of the mode scales up with the number of particles injected up to $\sim 10^{19}$ particles, after which complete cessation of the mode rotation generally leading to disruption has been observed. The decrease in rotation frequency as well as in the amplitude occurs within $\sim 1-2 m s$ of the application of a short gas puff pulse. The time interval between the gas-pulse application and reduction in amplitude of the mode also reduces with increase in amount of injected gas molecules. As these $m / n=2 / 1$ islands are observed to be rotating in the electron diamagnetic direction, the reduction in rotation frequency is most likely due to a change in the diamagnetic frequency caused by a reduction in the plasma temperature and a flattening of the density profile in the SOL/edge region due to the gas puffs. The density and floating potential fluctuations in the SOL/edge region are suppressed due to the gas-puffs. However, the observation of a decrease in mode amplitude along with the decrease in the rotation frequency is counter-intuitive to the analytical prediction of an increase in growth-rate of MHD modes with a decrease in the mode-rotation frequency. These experimental observations are well supported by simulations using the BOUT++ code [33]. The modification of the diamagnetic drift frequency in the edge and SOL region of ADITYA/ADITYA-U tokamak due to a gas puff (neutral injection) has been investigated using two-dimensional (2D) interchange plasma turbulence. The simulation results suggest $\sim 25-$ $65 \%$ reduction in the diamagnetic drift frequency due to an injection of $\sim 10^{18}$ neutral fuel particles in the edge region of ADITYA tokamak. 
In case of periodic gas puffs (gas - puff frequency, $f_{G P}, \sim 160-220 \mathrm{~Hz}$ ), the drift-tearing mode regains its original magnitude and rotation frequency, after the effect of the one gas puff is over. The sequence of events is repeated after each gas-puff. Hence a train of periodic gas puff pulses leads to a periodic modulation of the frequency and amplitude of the drift-tearing modes in ADITYA/ADITYA-U tokamak. This demonstration of alteration of drift-tearing modes using periodic gas-puffs may be useful for understanding the interaction of magnetic islands with the background plasma and may help in improving MHD control techniques.

The paper is organized as follows. Section 2 presents the experimental setup followed by experimental observations in section 3. The modelling results using BOUT++ code are presented in section 4 . The paper is concluded in section 5 .

\section{Experimental Set up}

The experimental observations and results presented in the paper are carried out in ADITYA tokamak [34], which is a medium sized ohmically heated air-core tokamak with a circular poloidal graphite limiter located at one toroidal location. It has a major radius $(\mathrm{R})$ of $75 \mathrm{~cm}$ and a minor radius (a) of $25 \mathrm{~cm}$ with toroidal magnetic $\left(B_{\phi}\right)$ field that varies from $\sim 0.9-1.26 T$. The typical discharge parameters are: plasma current $\left(I_{p}\right) \sim$ $80 \mathrm{kA}-170 \mathrm{kA}$, plasma duration $(t) \sim 200 \mathrm{~ms}-250 \mathrm{~ms}$, the chord-averaged central electron density $\left(n_{e}\right) \sim(2-5) \times 10^{19} \mathrm{~m}^{-3}$, chord averaged central electron temperature $\left(T_{e}\right) \sim 250 \mathrm{eV}-700 \mathrm{eV}$. The discharges analysed and reported in this paper have qedge in the range of $\sim 2.8-3.5$. This implies that the $\mathrm{q}=2$ rational surface is quite close to the plasma edge in these discharges. Further, the growth of islands, with the observed width of $m / n=2 / 1$ island $\sim 4-6 \mathrm{~cm}$ in these discharges, further brings them close to the edge. The base pressure is of $\sim 3 \times 10^{-8}$ Torr and hydrogen plasma is produced at a pressure $\sim 1-2 \times 10^{-4}$ Torr. Multiple hydrogen gas puffs are injected through a bottom port through a piezo-electric valve (500 SCCM at 100V), (poloidal angle, $\theta \sim 270^{\circ}$ and toroidal angle $\varphi \sim 90^{\circ}$ ) during the plasma current flat top by activating the piezo-electric valve by application of appropriate voltage pulses. These gas-feed pulses have been pre-fixed through a programmable voltage pulse generator prior to the initiation of the discharge, using a computer-controlled electronic circuit specially designed for gas feed system [35]. The magnitude and pulse-width of the voltage pulse are varied to inject a particular number of gas molecules in the plasma. The experiments are also repeated in ADITYA-U tokamak [36] which has the same machine parameters and similar plasma parameters. The only difference between the two machines is the limiter 
configuration, with ADITYA-U having a toroidal belt limiter whereas ADITYA has a poloidal ring limiter at one toroidal location.

A seven channel microwave interferometer is used for line averaged electron density measurement. The MHD oscillations are measured by a garland of 16 Mirnov coils distributed at equal angular separations in the poloidal direction at a single toroidal location in ADITYA. Each Mirnov coil has 35 turns and a cross-section of $2.9 \mathrm{~cm}^{-2}$. The ADITYA-U has two sets of a Mirnov garland placed at two different toroidal locations separated by 180 degrees each having 16 coils arranged equispaced along the poloidal periphery. An array of twelve surface barrier detectors (ORTEC, active area $\sim 50$ square mm, thickness $\sim 100 \mu \mathrm{m}$ ) are placed inside an imaging camera, to detect the soft X-ray emission from different chords fanned over the poloidal cross-section of the plasma. External magnetic sensors to measure loop voltage, toroidal plasma current, and plasma position have been used to determine standard plasma parameters. The data is recorded with a sampling frequency of $50 \mathrm{kHz}$ or $100 \mathrm{kHz}$ for all the diagnostics.

\section{Experimental Observations}

A representative discharge from ADITYA tokamak with 15 periodic gas-puff pulses applied during the plasma current flat-top at a frequency of $200 \mathrm{~Hz}$ is shown in figure 1 . Time evolution of loop voltage, plasma current, $\mathrm{H}_{\alpha}$ emission intensity and MHD activity acquired from one of the Mirnov coils (at $\theta \sim 320^{\circ}$ ) are shown in the figure. 


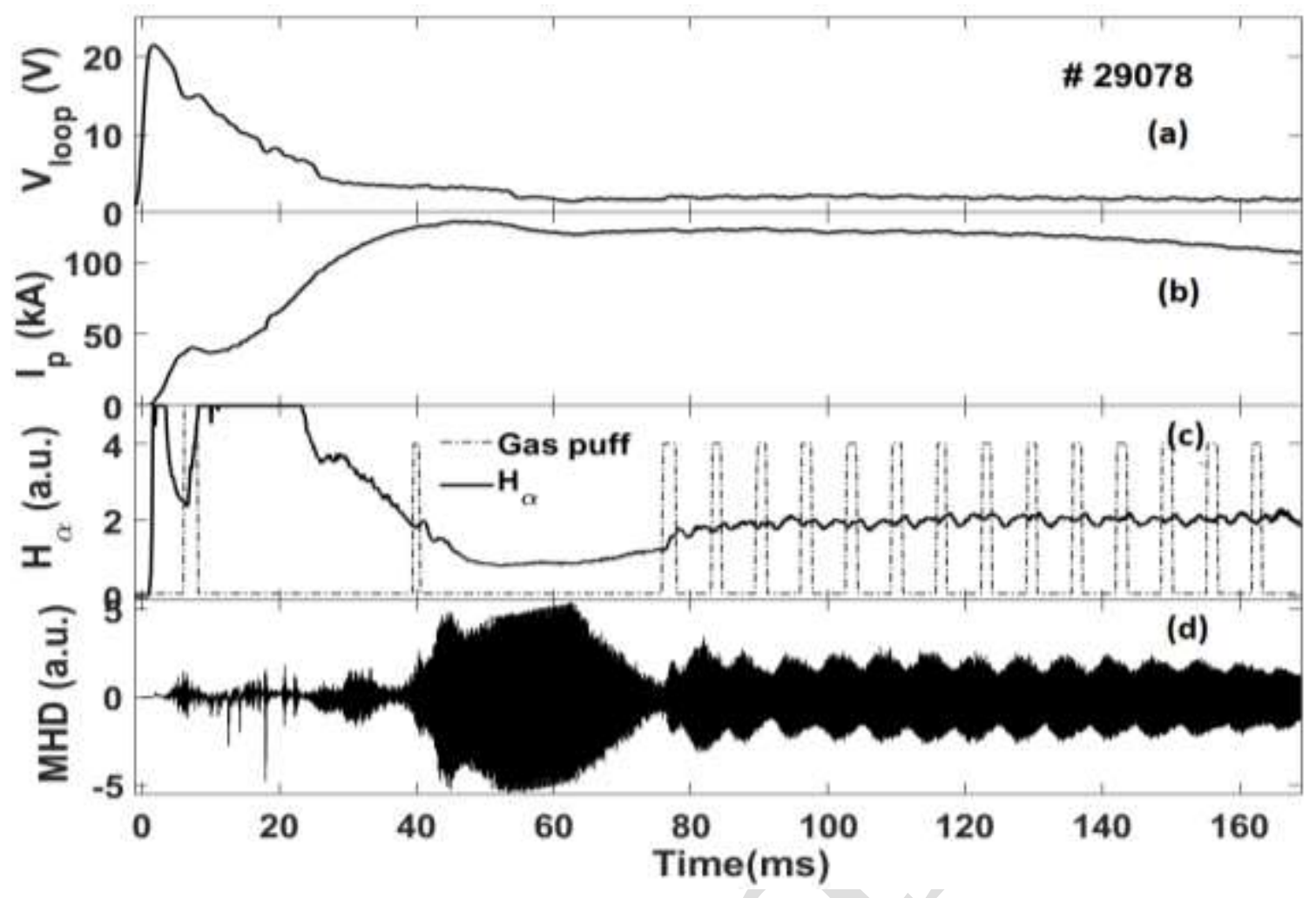

Figure 1: Plot showing the temporal evolution of (a) loop voltage, (b) plasma current, (c) $H_{\alpha}$ emission intensity and periodic gas puffs (d) MHD activity from shot \#29078.

The prime objective of fuel (hydrogen) gas-puffing during the discharge is to maintain the plasma density which is clearly reflected in the plasma particle inventory, both from the central line averaged and edge density measured by microwave interferometry and Langmuir probes respectively. Along with this, the gas-puffs trigger several other events in the discharge such as suppression of edge density and potential fluctuations, runaway electron ejection and changes in the characteristics of MHD modes [34] present in the discharges. The amount of injected gas is controlled by varying the amplitude and time duration (width), of the voltage pulse applied to the piezoelectric valve. In the discharges studied for this paper, the applied pulse-voltage has been varied between $\sim 4.5-6 \mathrm{~V}$ and the pulse duration has been varied from $\sim 1-2$ ms leading to the injection of $\sim 10^{17}-10^{18}$ hydrogen neutrals during each pulse. The delay between two pulses has been varied from $\sim 4-8$ ms over a large number of discharges. Experiments have been carried out by varying the number of gas-puff pulses and the time gap between the gas-puffs in different discharges.

\subsection{Impact of multiple periodic gas-puffs on rotation frequency of MHD modes}

The parameter $\widetilde{B_{\theta}} / B_{\theta}$ has been investigated during the current flat-top for $>3000$ discharges in ADITYA/ADITYA-U tokamak, since it is a measure of the island width/ MHD activity of 
a tearing mode [37]. To investigate the effect of gas puffs on the MHD mode characteristics in ADITYA and ADITYA-U tokamak discharges with moderate to strong $\mathrm{m} / \mathrm{n}=2 / 1$ and $3 / 1$ $\left(\widetilde{B_{\theta}} / B_{\theta}>0.5 \%\right)$ have been considered for the study. A large number of discharges in which these modes are present throughout the plasma current flat top with a nearly constant rotation frequency as well as amplitude have been analysed both in presence and absence of gas puffs. The mode structure analysis revealed the presence of $\mathrm{m} / \mathrm{n}=2 / 1$ and 3/1 MHD modes in ADITYA discharges [37] whereas in ADITYA-U mainly $m / n=2 / 1$ MHD mode has been found to be present as shown in figure 2(a). Figure 2(b) shows the direction of mode rotation which is in electron diamagnetic drift direction. The direction has been obtained by plotting the island structure at an interval of $0.02 \mathrm{~ms}$.
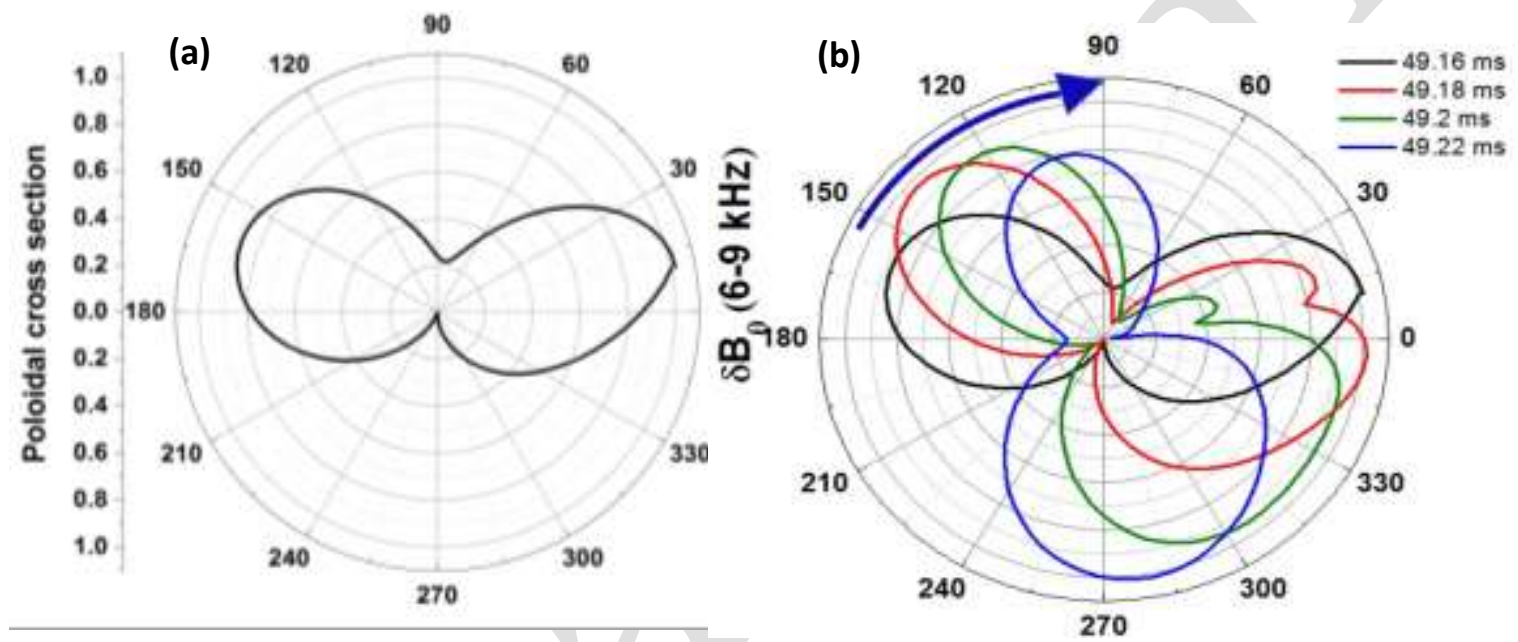

Figure 2: (a) Poloidal plot showing $m=2$ mode structure obtained by SVD analysis of 16 Mirnov coils in ADITYA-U (b) series of mode structure obtained at an interval of $0.02 \mathrm{~ms}$ showing clockwise rotation of $m=2$ mode.

The rotation frequencies of these MHD modes have been observed to be in the range of $\sim 5$ to $13 \mathrm{kHz}$, with a bandwidth of $\sim 1-2 \mathrm{kHz}$. The frequency spectra of Mirnov oscillations is obtained using the Fast Fourier Transform (FFT) algorithm and time evolution of the frequency spectrum is obtained by using frequency analysis function 'Specgram' in MATLAB. The Mirnov time-series data is sub-divided into multiple windows with 256 data points in each window (using Hanning window). FFT on each window is then carried to obtain the frequency amplitude spread for each window. The output graph is a color-map image with time and frequency on $\mathrm{X}$ and $\mathrm{Y}$ axis respectively and the magnitude at each frequency is reflected by a color code ('JET' type) with color 'red' representing the highest magnitude and blue being the lowest. Independent FFT analysis of Mirnov coil data segments at different time instants before, during and after gas puff pulse period has also been carried out in order to 
cross-check the frequency spectrum time profile obtained using the 'Specgram' routine. Frequency spectral analysis of Mirnov oscillations $\left(\dot{\mathrm{B}}_{\theta}\right)$ acquired by all Mirnov coils has been carried out for a large number of discharges using the above-mentioned method. The island structures are obtained by poloidal phase-plots of oscillations from all the 16 Mirnov coils corresponding to the dominant frequencies present in frequency spectra. The phase-plots are also used for obtaining the direction of rotation of the modes as presented later in the text.

In the absence of the gas-puffs, the temporal evolution of MHD activity remains nearly constant in its amplitude and frequency as shown in figure 3(a) and 3 (b) respectively.
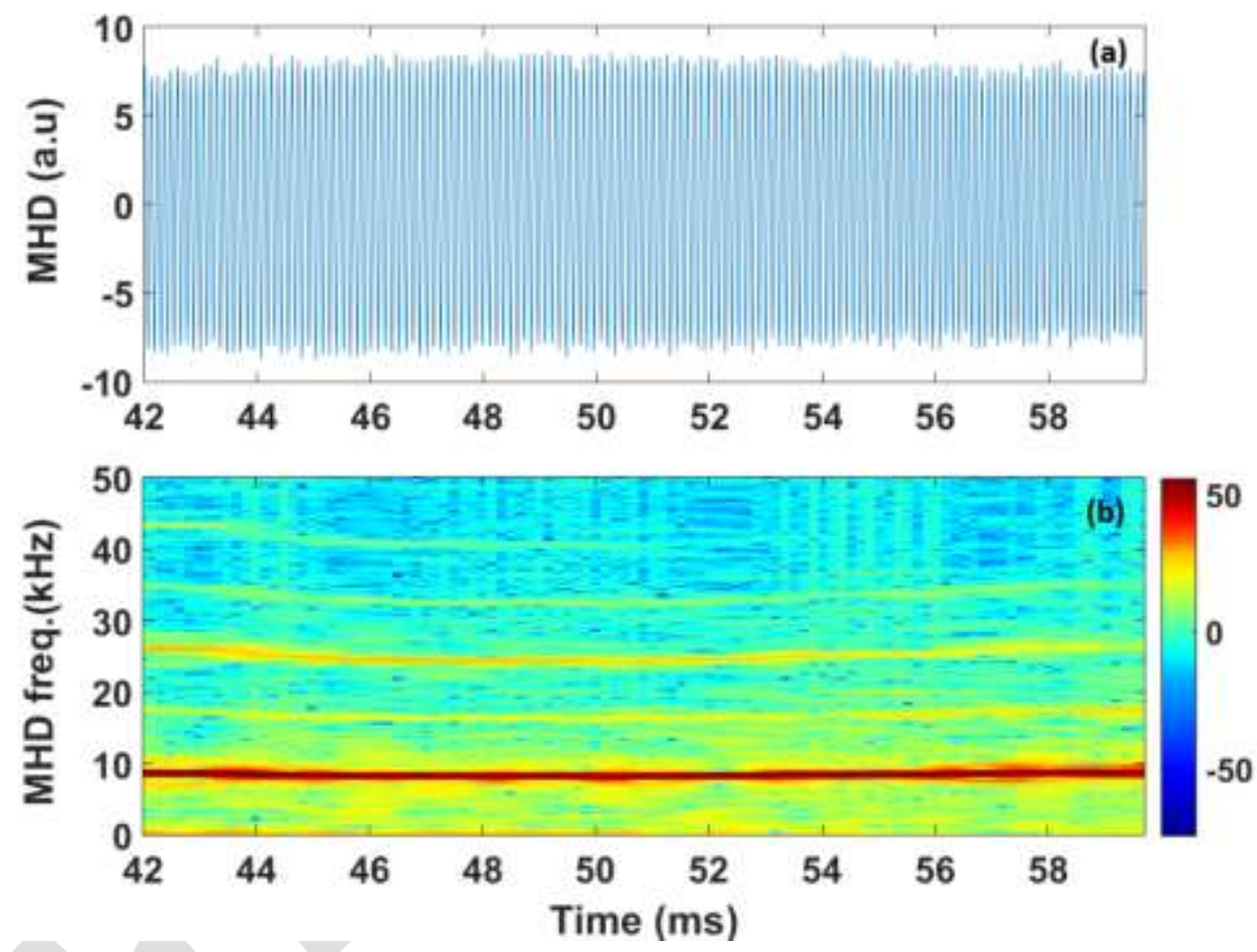

Figure 3: Plot showing (a) MHD amplitude and (b) its corresponding frequency spectrum of a discharge shot \#30629 without periodic gas puffs.

However, it can be clearly seen from figure 1, as soon as the train of gas-puffs starts at $\sim 76 \mathrm{~ms}$, a distinct periodic change strongly correlated with the gas-puff pulses appears in the temporal evolution of MHD amplitude. The MHD amplitude has been observed to decrease by 20-40 $\%$ within $1 \mathrm{~ms}$. The MHD activity remains at this reduced amplitude for the next $\sim 1-2 \mathrm{~ms}$ before increasing again until the next gas-puff pulse comes, whereupon the mode amplitude decreases again and the whole cycle is repeated after each gas-puff. Furthermore, the frequency spectral analysis of MHD activity from discharges with gas puff trains reveals that not only the amplitude of MHD activity is reduced due to the application of the gas-puff (figure 1(d)), the rotation frequency of these MHD modes also reduces after each gas-puff pulse. Figure 4 shows 
the time evolution of the frequency spectrum of MHD activity exhibiting the presence of multiple frequency bands in the specgram. The dominant frequency corresponds to the rotation frequency of $m / n=2 / 1$ mode. The other peaks are harmonics of the $m / n=2 / 1$ mode, explained in a separate publication [38].

It can be clearly seen from figure 4 that the dominant $\mathrm{m} / \mathrm{n}=2 / 1$ mode and its harmonics rotate with constant frequency $\sim 8 \mathrm{kHz}, 16 \mathrm{kHz}$ and $24 \mathrm{kHz}$ respectively during the plasma current flat-top, in absence of gas-puff..

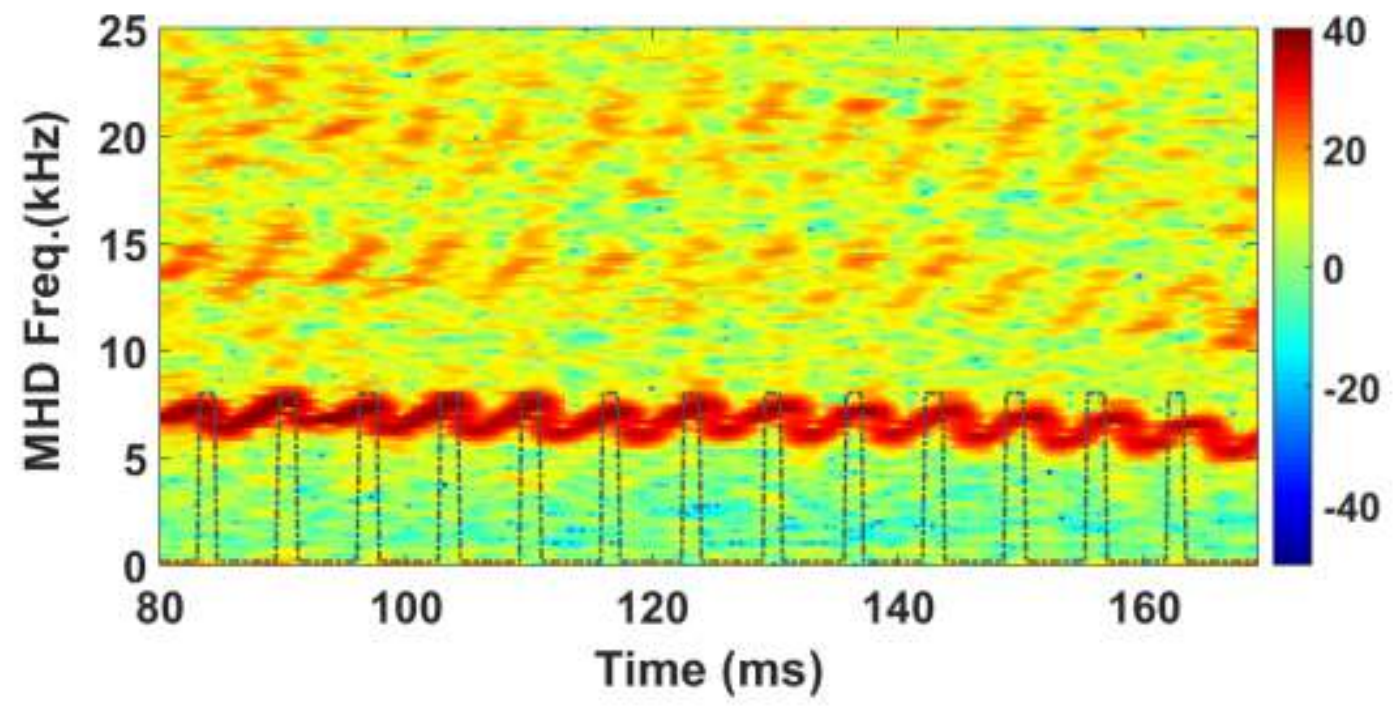

Figure 4: Plot showing frequency MHD modulation correlated with gas puffs specgram of Mirnov data from shot \#29078.

After the application of each gas-puff the mode rotation frequency of the $\mathrm{m} / \mathrm{n}=2 / 1$ mode decreases by $\sim 2 \mathrm{kHz}$ within $\sim 1 \mathrm{~ms}$ after the application of the gas puff pulse. The mode rotates at $\sim 6 \mathrm{kHz}$ under the influence of the gas-puff pulse for next $\sim 2-3 \mathrm{~ms}$ and thereafter regains and maintains its initial value of $\sim 8 \mathrm{kHz}$ until the next gas puff pulse reduces its frequency again. The whole cycle is repeated after each gas puff pulse. The higher frequency harmonics are also influenced in a similar fashion as the frequency of the first harmonics $(\sim 16 \mathrm{kHz})$ decreases by $\sim 4 \mathrm{kHz}$, after the gas-puff pulse. The above-mentioned observations clearly establish that the amplitude and rotation frequency of MHD modes are influenced by injecting an appropriate amount of fuel (hydrogen) gas in plasma discharges of ADITYA and ADITYA-U tokamak. To investigate further the nature of the interaction between the gas-puff and the MHD modes, the amount of puffed gas has been varied either on 
a shot-to-shot basis or during the course of a single discharge. The decrement in rotation frequency of the MHD modes appeared to be higher with higher amount of injected neutrals. The decrement in rotation frequency of the MHD modes has been plotted with respect to the amount of neutrals injected in figure 5. Each data point corresponds to the decrement in rotation

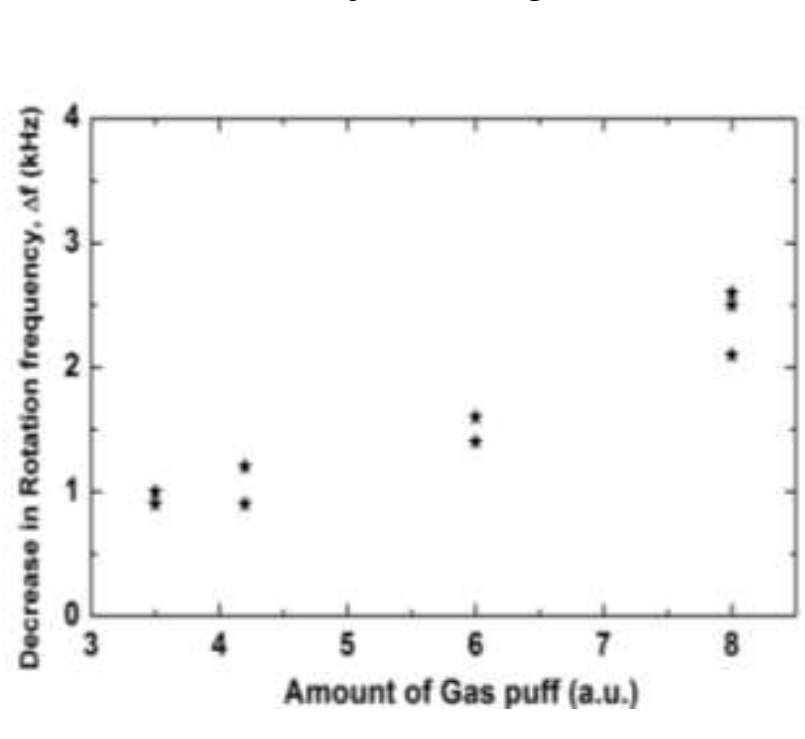

Figure 5: Decrement in 2/1 mode rotation frequency after a gas puff plotted with respect to the amount of gas puff injected for different discharges.

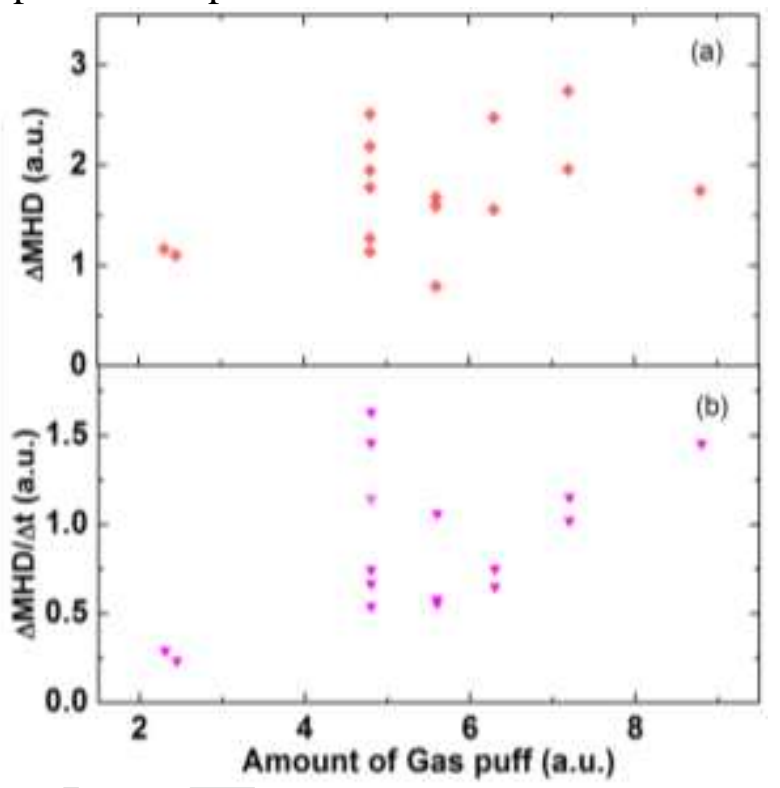

Figure 6:(a) Decrease in MHD amplitude and (b) Rate of change of MHD amplitude plotted with respect to the amount of gas puff injected for several discharges from ADITYA.

frequency after gas puff in during the current flat-top of different discharges with different amount of injection of gas puff. The figure shows that the decrease in the rotation frequency is almost proportional to the amount of neutrals injected up to $\left(\sim 10^{19}\right)$ neutrals injected for these discharges beyond which they induce disruptions. Similarly, the decrement in the MHD amplitude has also been found to be increasing systematically as shown in figure 6 (a). Figure 6(b) further shows that the rate of decrease of the amplitude is also increased with increasing the amount of injected molecules, i.e., the mode amplitude decreases with a faster rate after the application of the gas-puff pulse as the amount of injected neutrals is increased.

For the discharges presented in this paper, the toroidal magnetic field, $B_{\phi}$, is in the anticlockwise direction and the plasma current is in the clockwise direction as viewed from the top, both in ADITYA and ADITYA-U [36]. Hence, the electron diamagnetic drift has a poloidally clockwise direction. Before the gas injection, the observed coherent $m / n=2 / 1 \mathrm{MHD}$ modes are found to be rotating in a poloidally clockwise direction. The direction of rotation of these modes is obtained by plotting the filtered Mirnov fluctuations at the coherent frequency $\sim 5-14 \mathrm{kHz}$ of the dominant $\mathrm{m} / \mathrm{n}=2 / 1$ mode for all the 16 coils. Figure 7 (a) and (b) show 
the time evolution of raw Mirnov fluctuations at different poloidal angle arranged in a clockwise direction starting from outboard $0^{\circ}$ and the corresponding contour plot using all 16 Mirnov coils. From these plots, it is concluded that the observed $m / n=2 / 1$ MHD modes are rotating in electron diamagnetic direction prior to the gas puff.

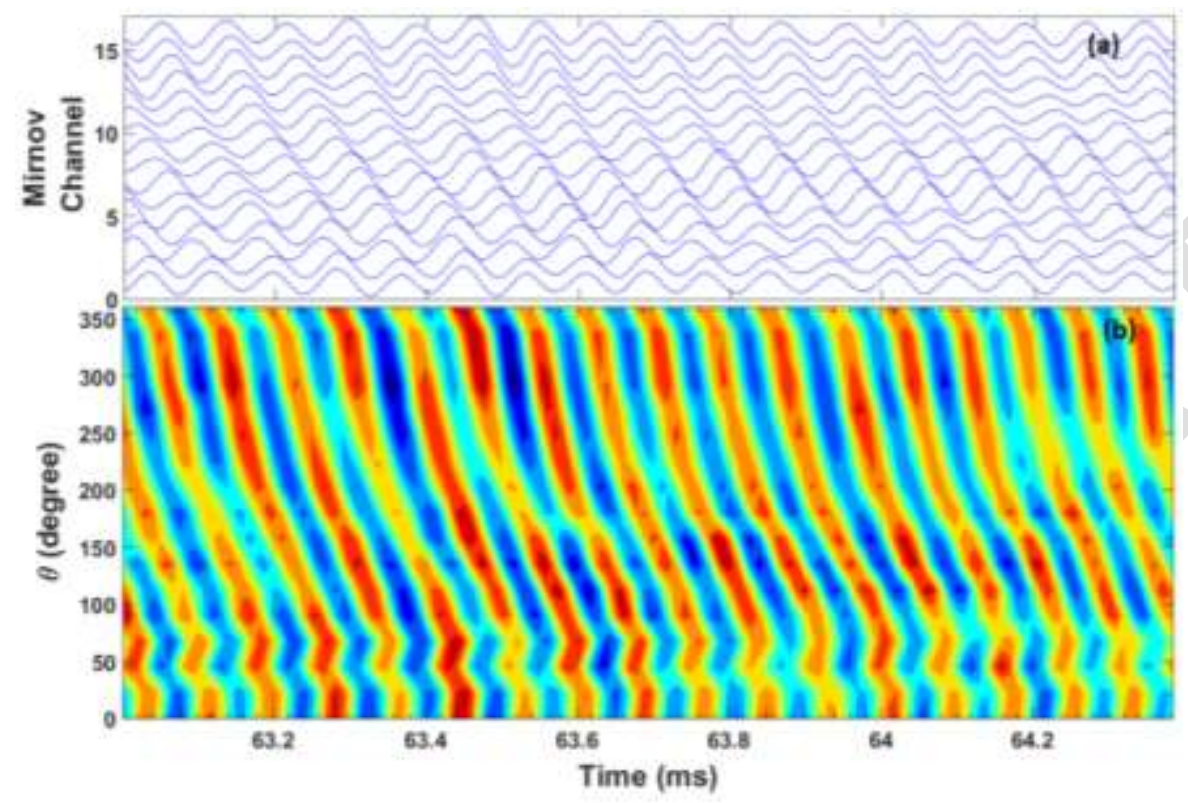

Figure 7:(a) Poloidal magnetic fluctuations from 16 Mirnov coils (b) and their Contour plot

It has also been observed that the amplitude of the $\mathrm{m} / \mathrm{n}=2 / 1$ mode depends on the mode rotation frequency. The amplitude of the $\mathrm{m} / \mathrm{n}=2 / 1$ mode $\left(\delta B_{\theta}\right)$ is plotted against the mode rotation frequency from several ADITYA discharges in figure 8.

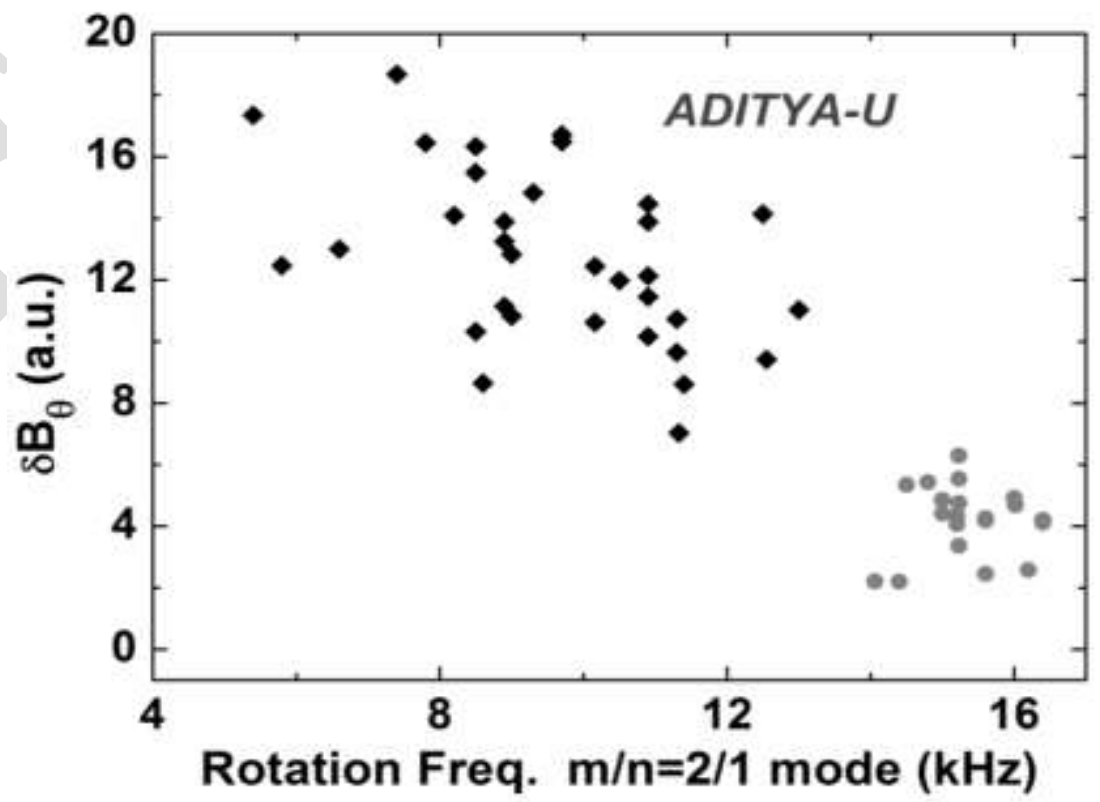


Figure 8: Plot showing the MHD amplitude $\left(\delta B_{\theta}\right)$ with respect to the rotation frequency of $m / n=2 / 1$ mode obtained during the current flat top of different discharges in ADITYA_U

The figure shows that the amplitude of the mode decreases with increasing mode rotation frequency. The observed rotation frequency of the dominant $\mathrm{m} / \mathrm{n}=2 / 1$ mode $\sim 5-10 \mathrm{kHz}$ matches well with the diamagnetic frequency calculated using

$\omega_{e}^{*}=\frac{k_{y} T_{e}}{e B L_{n}}$

with electron temperature $T_{e} \sim 50 \mathrm{eV}$ and density scale length $1 / L_{n} \sim 4 \mathrm{~cm}^{-1}$ measured in the edge region $[28,39]$ in absence of the gas-puff as shown in figure 9.

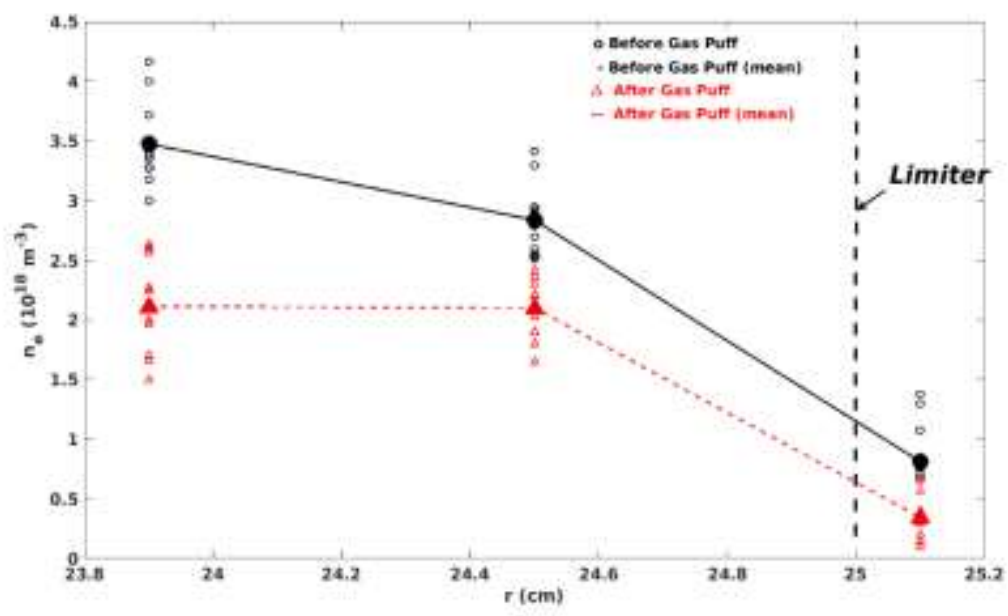

Figure 9: Radial profile of density measured by 3 Langmuir probes placed at different radial locations in the edge region. The light-coloured open symbols are the measurements from different gas-puff cycles and from different discharges. The big, brighter, solid-symbol represents the average of all measurements at a single radius. Open and filled circles with solid line: before gas-puff pulse; Open and filled triangles with dotted line: after the gas-puff pulse.

Furthermore, coherent peaks at a frequency similar to that of the dominant $m / n=2 / 1$ mode are observed in the frequency spectra of electron density fluctuations and floating potential fluctuations both in the edge and SOL region of ADITYA/ADITYA-U tokamak as shown in figure 10 . 


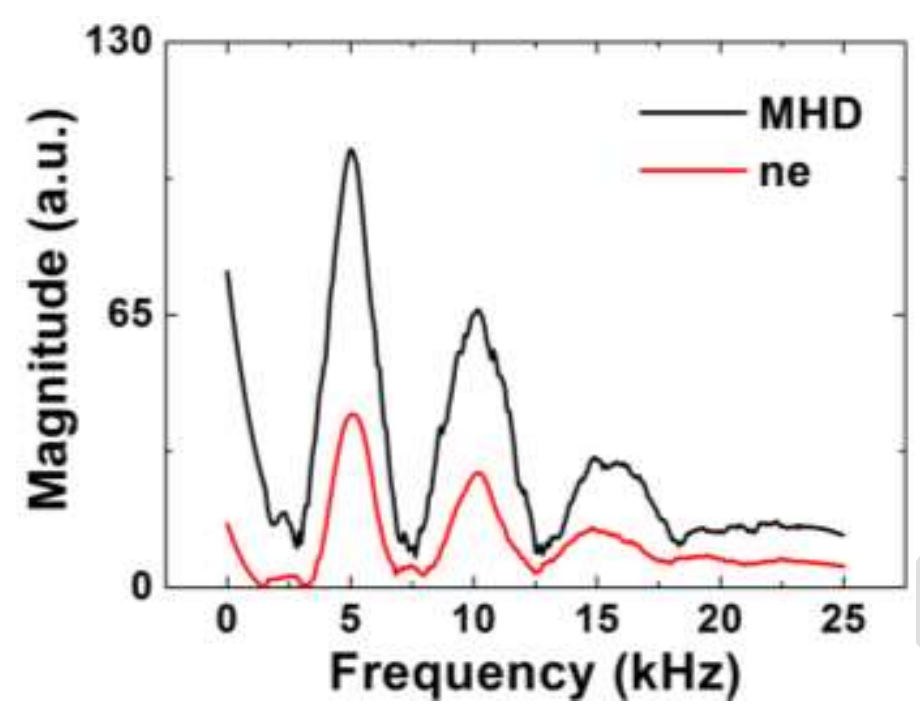

Figure 10: Frequency vs. magnitude plot obtained by FFT of MHD (black) and density(red) data for shot \#29078

These observations suggest that the observed MHD modes fall in the drift-tearing mode category and rotate with a real frequency of $\sim 5-14 \mathrm{kHz}$ in the electron diamagnetic direction. We note here that these observations are repeated for a good number of discharges prior to gaspuff pulse injection.

When the neutrals are injected through a gas-puff pulse at the current flat-top in the discharges with existing high-amplitude $\left(\widetilde{B_{\theta}} / B_{\theta}>5 \%\right) \mathrm{m} / \mathrm{n}=2 / 1$ MHD modes, a reduction in the frequency of rotation of these modes along with a reduction in the amplitude of the MHD fluctuations have been observed. As mentioned earlier in the text, the rotation frequency of the MHD island in the laboratory frame is a combination of the plasma rotation frequency and the phase velocity of the island in the plasma frame. Hence, the rotation frequency downshifts after a gas-puff pulse may either be due to the reduction in the frequency of the electron diamagnetic drift or that of the $\boldsymbol{E} \times \boldsymbol{B}$ plasma rotation. Since the radial electric field $\left(E_{r}\right)$ has a radially outward direction, the resultant $\boldsymbol{E} \times \boldsymbol{B}$ rotation would be in the poloidally anticlockwise direction. Figure 11 shows the direction of different drifts in ADITYA and ADITYA-U. 


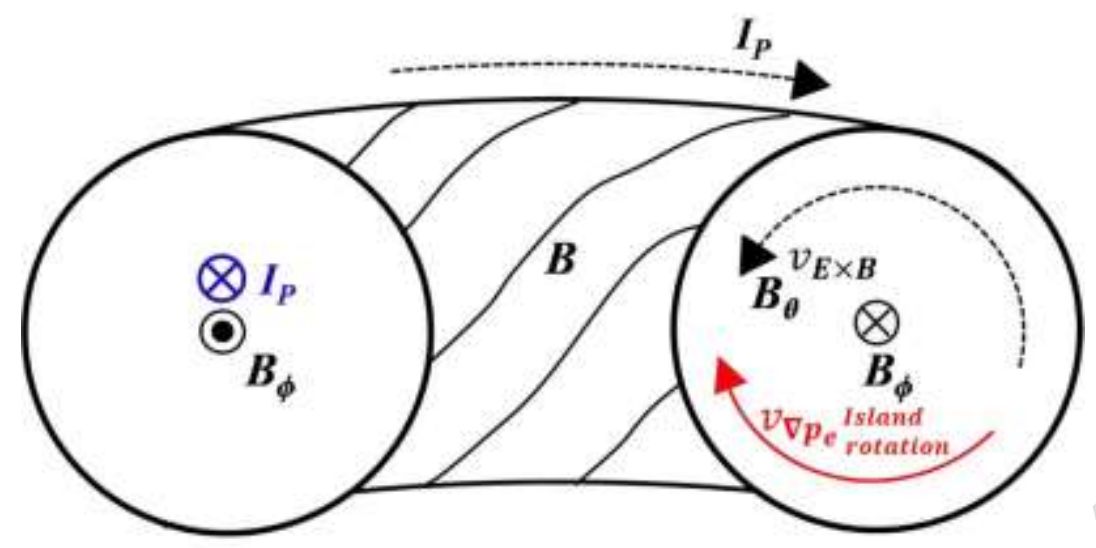

Figure 11: Schematic of ADITYA showing the toroidal direction of plasma current, toroidal magnetic field and the direction of electron diamagnetic drift, $E \times B$ drift, and the island rotation.

With the introduction of the gas puff, the island continues to rotate in the electron diamagnetic drift direction but with a reduced frequency, which indicates that the diamagnetic drift appears to have the dominant influence on the mode rotation in both ADITYA and ADITYA-U. The $E \times B$ rotation also reduces, due to a reduction in the potential fluctuation (and hence $E$ ), as has been noted before in the edge region of the ADITYA tokamak [40]. However, the reduction in $E \times B$ cannot decrease the mode rotation frequency as it is in the opposite direction.

The radial profile of electron density in the edge/SOL region is measured using Langmuir probes. The radial profile of density at two time intervals, before and after the gas-puff are shown in figure 9. The light-coloured open circle and open triangle symbols in figure 9 represent the measurements at different radial locations from different gas-puff cycles and from different discharges before and after the gas-puff pulse injection respectively. The bigger, brighter, filled-symbols (circle and triangle for before and after gas-puff respectively) represent the average of all measurements at a single radius. It can be clearly seen from the figure that after the application of the gas puff the density profile in the edge/SOL region flattens, i.e., the density gradient in the edge/SOL region decreases after the gas-injection from its value in absence of the gas-injection. Earlier experiments in ADITYA have also demonstrated that neutral injection reduces the edge temperature $\left(T_{e}\right)$ and increases the edge density scale length $\left(L_{n}\right)$. Similar observations of reduction in edge/SOL temperature and flattening of density profiles have also been reported from previous experiments in other tokamaks. As the electron temperature and the density gradient decrease with the application of the gas puff, the diamagnetic drift frequency also decreases according to the above mentioned equation of diamagnetic drift frequency. The decrease in the drift frequency is further corroborated with the observation of a significant reduction in the density and floating potential fluctuations 
measured with Langmuir probes in SOL region of ADITYA-U tokamak after the gas injection as shown in figure 12 (a) and (b) respectively.

(a)

(b)

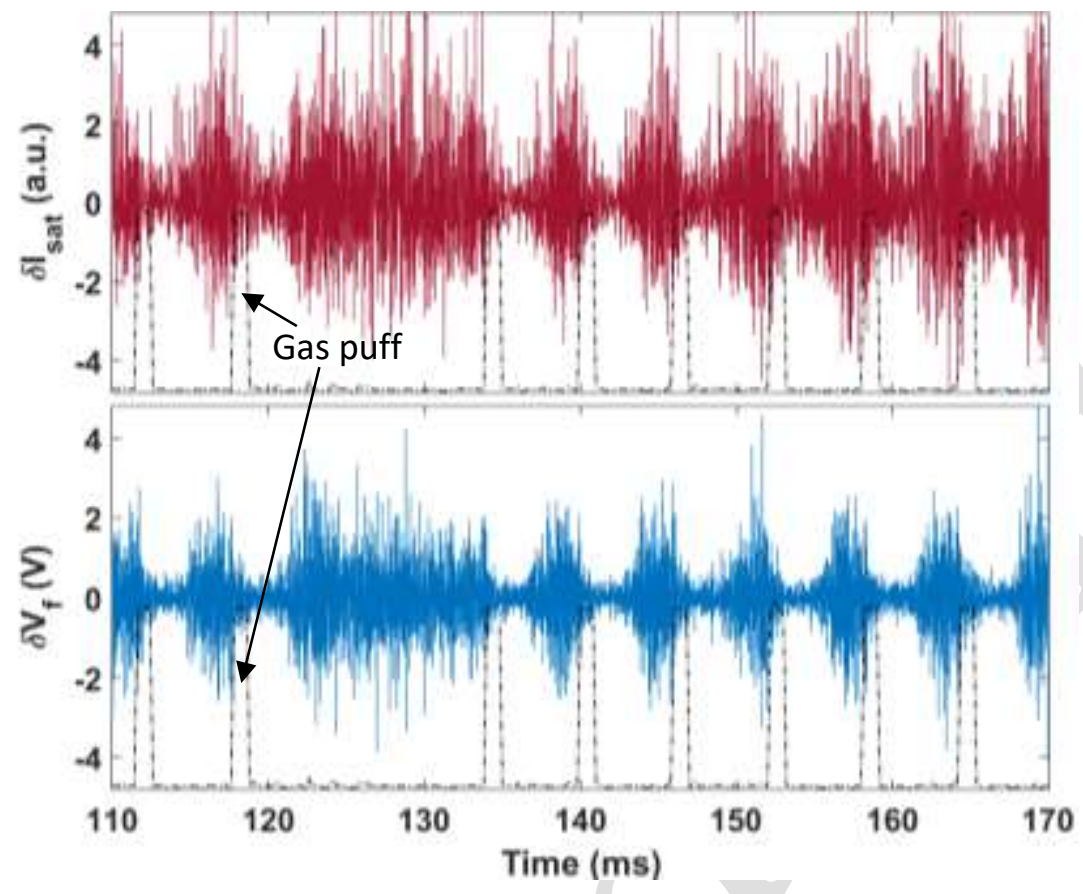

Figure 12: Plot showing (a) edge density fluctuations filtered from Ion saturation current signal and (b) potential fluctuations acquired from Langmuir probes in ADITYA-U tokamak, exhibiting fluctuation suppression after each gas puff.

As the diamagnetic drift frequency reduces with the application of short gas-puff pulse, the rotation frequency of the $\mathrm{m} / \mathrm{n}=2 / 1$ drift-tearing mode also decreases, which fairly describes our experimental observations. The argument attributing the observed downshift of the rotation frequency of the island to be due to the reduction in drift frequency $\left(\omega^{*}\right)$ is further substantiated by changing the amount of injected gas in one gas-puff pulse. As the amount of the injected gas is increased the change (decrease) in electron temperature and density gradient increases in the edge/SOL, leading to a further decrease in island rotation frequency as shown in figure 5. As mentioned earlier, the discharges analysed have qedge values in the range of $\sim 2.8$ to 3.5 . The discharge \#29078 for which the parameters are shown in figure 1, has a qedge $~ 2.8$. Having qedge $\sim 2.8-3.5$ implies that the $\mathrm{q}=2$ rational surface is quite close to the plasma edge in these discharges. Furthermore, the growth of islands, with the observed width of $m / n=2 / 1$ island $4-6 \mathrm{~cm}$ in these discharges, brings them even closer to the edge. Due to the relatively low edge temperature and density of the ADITYA discharges the gas due to gas-puff penetrates well inside the edge region, modifying the density gradient there, hence affecting the dynamics of the $\mathrm{m} / \mathrm{n}=2 / 1$ mode in ADITYA. 
Interestingly in the majority of discharges, where a train of gas-puff pulses is applied with a repetition frequency of $\sim 200 \mathrm{~Hz}$ (time period $\sim$ global confinement time of the plasma), the rotation frequency of the $m / n=2 / 1$ mode and its harmonics are observed to be modulated with the rotation frequency decreasing after each gas puff and regaining its prior-to-gas-puff value once the effect of gas-puff ceases.. The short gas-puff pulse reduces the temperature and density gradient in the edge/SOL region for a short duration and after the neutrals are completely ionized the edge/SOL region is heated again in presence of the loop voltage and $\omega^{*}$ increases again. The modulation of rotation frequency and the amplitude of the $2 / 1$ mode and its harmonics continue throughout the plasma current flat-top with the application of a train of short gas-puff pulses. Furthermore, these trains of gas-puff pulses have been observed to affect the global plasma parameters including the energy confinement favourably as improvement in these parameters are observed with the application of gas-puff pulses [34]. If instead of a short gas-puff pulse $(<2 \mathrm{~ms})$, a gas-puff pulse of larger duration is applied then although the rotation frequency is reduced after the pulse, it regains its prior-to-gas-puff value even though the gas pulse is still on. The gas injection can also influence the torque and thus the tearing mode stability parameters $\left(\Delta^{\prime}\right)$. The gas injection $\left(\sim 10^{21}\right.$ neutrals $)$ is known to drive a radial current and the torque associated with this radial current induces rotation which modifies the transport leading to modifications in the MHD island dynamics [41] However, in our experiments the amount of gas injection is very small $\left(\sim 10^{17}-10^{18}\right.$ neutrals $)$ and hence the magnitude of radial currents is significantly low and unlikely to have a major influence on the transport properties.

\subsection{Impact of multiple periodic gas-puffs on amplitude of MHD modes}

The other interesting observation with the short gas-puff pulse application is the reduction of the amplitude of the $\mathrm{m} / \mathrm{n}=2 / 1 \mathrm{MHD}$ mode when the mode rotation frequency decreases. We note here that the observed reduction in amplitude of the mode is not due to measurement errors. As the voltage induced in the Mirnov coils depends on the rotation frequency of the modes $\left(V_{\text {Mirnov }}=n * A *\left(\frac{d B_{\theta}}{d t}\right)\right.$, with $n=$ number of turns in Mirnov coil and $A=$ crosssection area of the coil), with the observed reduction in the mode frequency after the gas-puff pulse, the amplitude of the voltage induced in the Mirnov coil should decrease. However, the observed decrease in amplitude of the voltage is much larger than that calculated by taking a rotation frequency reduction of $\sim 2-4 \mathrm{kHz}$ due to the gas injection and hence is not an artefact of the measurement. 
Such a behaviour of the growth rate (and corresponding mode amplitude) with the real frequency has been predicted by linear stability studies [5] to occur over a limited regime for $\omega^{*}>\gamma_{T}$ (where $\gamma_{T}$ is the growth rate of the tearing mode). For the relatively lower temperature discharges of ADITYA/ADITYA-U (with normalized resistivity [5], $\eta \sim 10^{-6}$ and $\left.\gamma_{T} \lesssim 1 \mathrm{kHz}[13]\right)$, the values of $\omega^{*} / \gamma_{T}$ remains on the side of the transition point where the $\gamma / \gamma_{T}$ is an increasing function of $\omega^{*} / \gamma_{T}$. However a linear theory of the drift-tearing mode is not adequate to explain our observations since the mode is likely to be in the nonlinear regime. The effect could be arising from modifications in the value of $\Delta^{\prime}$ due to the change in the edge profiles caused by the gas puff. Additionally nonlinear changes in the island size induced by diamagnetic effects could also be at play [42].

\section{Modelling using BOUT++}

To further understand the observations mentioned in the above sections, the effect of short gaspuff pulses on the ADITYA/ADITYA-U tokamak plasma discharges has been simulated using the BOUT++ code $[33,43]$. In edge and SOL regions, in the presence of the ionizing electrons and the neutral gas, a number of atomic and molecular reactions such as electron impact molecular dissociations, ionization, molecular ionic dissociations, and ionic recombination, etc. occur. Details of these reactions are given in Refs.[44,45] which indicate that the plasma in the SOL region consists of electrons, $\mathrm{H}^{+}, \mathrm{H}^{+2}$, and $H_{2}$ molecules mainly. Details of the model equations and normalization of each parameter have been given in [33]. The main equations are as follows:

$$
\begin{aligned}
\frac{d n}{d t}-D \nabla_{\perp}^{2} n+g & \left(T_{e} \frac{\partial n}{\partial y}+n \frac{\partial T_{e}}{\partial y}-n \frac{\partial \phi}{\partial y}\right) \\
& =\xi_{i o n}\left(T_{e}\right) n N+\xi_{i o n}^{a}\left(T_{e}\right) n N+\chi_{e d g e} \chi_{0} T_{e 0}^{-\frac{3}{2}}\left\{\phi-T_{e} \ln (n)\right\}-\sigma_{0} n \sqrt{T_{e}} e^{\Lambda-\frac{\phi}{T_{e}}} \\
& +S_{n}
\end{aligned}
$$

$$
\begin{aligned}
\overrightarrow{\nabla_{\perp}} \cdot\left(n \frac{\partial}{\partial t} \overrightarrow{\nabla_{\perp}} \phi\right) & -v \nabla_{\perp}^{4} \phi+g\left(T_{e} \frac{\partial n}{\partial y}+n \frac{\partial T_{e}}{\partial y}\right) \\
& =n \sigma_{s o l} \sigma_{0} \sqrt{T_{e}}\left(1-e^{\Lambda-\frac{\phi}{T_{e}}}\right)+\chi_{e d g e} \chi_{0} T_{e 0}^{-\frac{3}{2}}\left\{\phi-T_{e} \ln (n)\right\}-\left(v_{i m} N\right. \\
& \left.+v_{i a} N_{a}\right) \nabla_{\perp}^{2} \phi
\end{aligned}
$$

$$
\begin{aligned}
\frac{d T_{e}}{d t}-k_{e} \nabla_{\perp}^{2} T_{e} & +\frac{2}{3} g\left(\frac{7}{2} T_{e} \frac{\partial T_{e}}{\partial y}+\frac{T_{e}^{2}}{n} \frac{\partial n}{\partial y}-T_{e} \frac{\partial \phi}{\partial y}\right) \\
& =-\frac{2}{3} g f_{c} \xi_{\text {eff }}^{\text {loss }} N E_{\text {loss }}--\frac{2}{3} f_{E} \sigma_{\text {sol }} \sigma_{0} T_{e}^{-\frac{3}{2}} e^{\Lambda-\frac{\phi}{T_{e}}},
\end{aligned}
$$




$$
\frac{\partial N}{\partial t}-\overrightarrow{\nabla_{\perp}} \cdot\left(D_{n}(n) \overrightarrow{\nabla_{\perp}} N\right)=\xi_{i o n}^{N} n N+f_{E} \sigma_{s o l} \sigma_{0} n \sqrt{T_{e}}
$$

where $n, n_{2}, \phi, T_{e}$, and $N$ indicate electron density, $H_{2}^{+}$density, potential, electron temperature, and neutral gas density, respectively. The contribution of hot or energetic ions has been ignored and each ion species is assumed to be collected at the limiter plate with a common sound speed. It is to be noted that for quantitative comparison with the experimental results the above equations should be solved in the toroidal coordinate system using a kinetic description of the neutral gas. But here a simple 2D rectangular coordinate system and a fluid description of the neutral gas have been used as we are interested in the qualitative matching of the experimental observations and results. Equations (1) - (5) have been solved numerically using BOUT++ code. The above equations are solved using normalised neutral source rate $S_{n}=1 \times 10^{-4}$ and varying the magnitude from $S_{N 0}=1-6 \times 10^{-5}$. It is to be noted that $S_{n}=$ $1 \times 10^{-4}$ corresponds to the injection of $\sim 3 \times 10^{20}$ particles from the core plasma to the edge-SOL regions and $S_{n}=1 \times 10^{-5}$ corresponds to the injection of $\sim 3 \times 10^{19}$ particles in the outermost SOL region through an area of $\sim 1 \mathrm{~m}^{2}$ in the $y z$ plane. In the simulation, the contribution of hot or energetic ions has been ignored and each ion species is assumed to be collected to the limiter plate with their own Bohm sheath criteria. A finite-difference in the radial $\mathrm{x}$-direction and FFT in the poloidal y-direction have been used for spatial integration. The time integration has been done using stiffly stable fourth-order Adams-Bashforth predictor-corrector method. Details of the input parameters as given in the model equations can be found in Ref. [33,43].

Figure 13 shows the simulated variation of $\omega^{*}$ (normalized to ion gyro-frequency) versus the distance from separatrix (normalized to ion gyroradius) in absence and presence (gas injection rate of $\sim 5 \times 10^{20}$ molecules per second as in the case of experiments) of injected gas. 


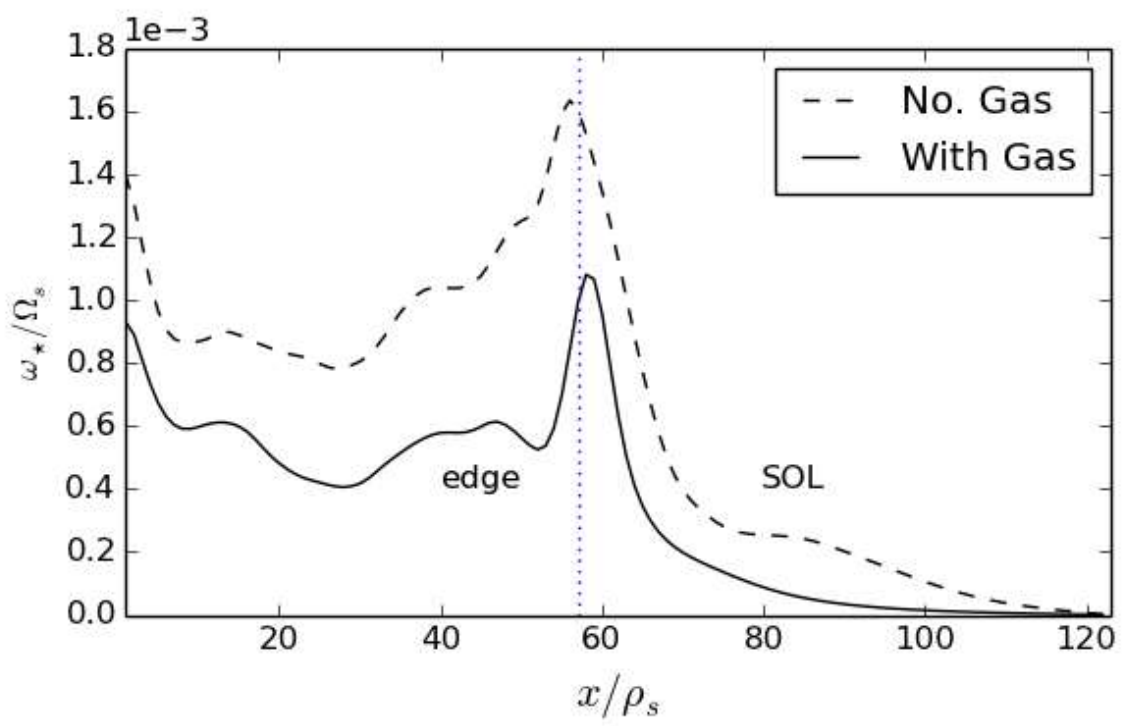

Figure 13: BOUT++ Simulation results comparing drift frequency in edge/SOL of ADITYA with and without gas puff.

The figure clearly shows that $\omega^{*}$ reduces significantly when neutral gas is injected and thereby suggests that the observed decrease of MHD mode rotation frequency is due to the reduction in $\omega^{*}$. A maximum of $60 \%$ reduction has been found in the $\omega^{*}$, from the numerical investigations with an injection of $\sim 10^{17}-10^{18}$ neutrals in the edge/SOL region. The almost linear increase in the decrement of $\omega^{*}$ with the increase in the amount of injected gas molecules observed in experiments (figure 5) is also reproduced in the simulations as shown in figure 14.

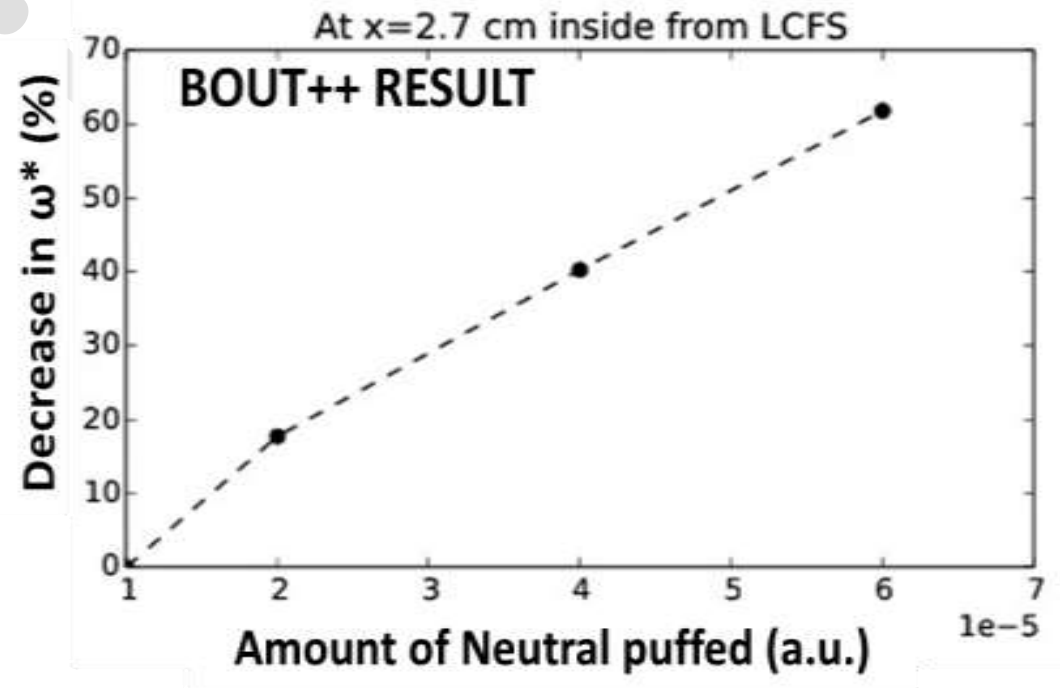

Figure 14: Plot showing simulation results for decrease in $\omega^{*}$ with increase in the amount of neutrals injected. 


\section{Conclusion}

With the application of short gas-puff pulses, the rotation frequency as well as the magnitude of the drift-tearing mode, existing prior to the gas-puff, is observed to be decreased systematically in typical discharges of ADITYA/ADITYA-U Tokamak. A train of periodic short gas-puff pulses applied at the plasma current flat-top leads to periodic modifications of the mode rotation frequency and magnitude of drift-tearing modes. Mode analysis revealed that the dominant $\mathrm{m} / \mathrm{n}=2 / 1$ mode and its harmonics rotate in the electron diamagnetic direction prior to the gas puff with a rotation frequency $\sim-14 \mathrm{kHz}$. After the application of short fuel (hydrogen) gas-puff pulse injecting $10^{17}-10^{18}$ molecules, the $2 / 1$ mode continues to rotate in the electron diamagnetic direction, however with a decreased rotation frequency and the amplitude of the mode is also observed to be reduced. The decrease in the rotation frequency, as well as the amplitude of the MHD mode, is found to be proportional to the number of neutrals injected until the gas injection disrupts the plasma. As the electron temperature decreases and the density scale length increases with the gas injection, the drift wave frequency also decreases leading to a reduction in the observed rotation frequency of the $\mathrm{m} / \mathrm{n}=2 / 1$ mode and its harmonics. Although the $\boldsymbol{E} \times \boldsymbol{B}$ plasma rotation also reduces with the gas-puff, however, it may not be causing the decrease in the mode rotation frequency as it is in the opposite direction to the direction of the mode rotation. The BOUT++ code simulation corroborates the experimental results of plasma pressure profile modifications in the edge/SOL region, leading to a reduction in diamagnetic drift frequency, subsequently reducing the rotation frequency of drift-tearing mode. Interestingly, the amplitude of the mode is also observed to be reduced after the gas puff along with the reduction in rotation frequency. A current profile modification, affecting the $\Delta^{\prime}$, may be taking place in the edge region due to the injection of the short gaspuff pulse. Stabilizing effect of $\Delta^{\prime}$, due to the current profile modification by short gas-puff injection may very well lead to decrease of saturation amplitude of the tearing modes as observed in the experiments. 


\section{References:}

[1] Hender T ., Wesley J ., Bialek J, Bondeson A, Boozer A ., Buttery R ., Garofalo A, Goodman T ., Granetz R ., Gribov Y, Gruber O, Gryaznevich M, Giruzzi G, Günter S, Hayashi N, Helander P, Hegna C ., Howell D ., Humphreys D ., Huysmans G T ., Hyatt A ., Isayama A, Jardin S ., Kawano Y, Kellman A, Kessel C, Koslowski H ., Haye R J La, Lazzaro E, Liu Y ., Lukash V, Manickam J, Medvedev S, Mertens V, Mirnov S ., Nakamura Y, Navratil G, Okabayashi M, Ozeki T, Paccagnella R, Pautasso G, Porcelli F, Pustovitov V ., Riccardo V, Sato M, Sauter O, Schaffer M ., Shimada M, Sonato P, Strait E ., Sugihara M, Takechi M, Turnbull A ., Westerhof E, Whyte D ., Yoshino R, Zohm H and Group the I M D and M 2007 Chapter 3: MHD stability, operational limits and disruptions Nucl. Fusion 47 S128-202

[2] Fitzpatrick R 2011 Fundamentals of Magnetic Island Theory in Tokamaks Fusion Sci. Technol. $59625-625$

[3] Bussac M N, Edery D, Pellat R and Soule J L 1978 Stabilization of the Linear Drift Tearing Mode by Coupling with the Ion Sound Wave Phys. Rev. Lett. 40 1500-3

[4] Fitzpatrick R and Waelbroeck F L 2008 Drift-tearing magnetic islands in tokamak plasmas Phys. Plasmas 15

[5] Biskamp D 1978 Drift-tearing modes in a tokamak plasma Nucl. Fusion 18 1059-68

[6] Lütjens H, Luciani J-F and Garbet X 2001 Curvature effects on the dynamics of tearing modes in tokamaks Phys. Plasmas 8 4267-70

[7] Kotschenreuther M, Hazeltine R D and Morrison P J 1985 Nonlinear dynamics of magnetic islands with curvature and pressure Phys. Fluids 28 294-302

[8] Volpe F A, Hyatt A, La Haye R J, Lanctot M J, Lohr J, Prater R, Strait E J and Welander A 2015 Avoiding Tokamak Disruptions by Applying Static Magnetic Fields That Align Locked Modes with Stabilizing Wave-Driven Currents Phys. Rev. Lett. 115 $1-5$

[9] Connor J W, Waelbroeck F L and Wilson H R 2001 The role of polarization current in magnetic island evolution Phys. Plasmas 8 2835-48

[10] White R B, Monticello D A, Rosenbluth M N and Waddell B V. 1977 Saturation of the tearing mode Phys. Fluids 20 800-5 
[11] Liu T, Wang Z X, Hu Z Q, Wei L, Li J Q and Kishimoto Y 2016 On the threshold of magnetic island width in nonlinear mutual destabilization of tearing mode and ion temperature gradient mode Phys. Plasmas 23 1-7

[12] Scott B D, Hassam A B and Drake J F 1985 Nonlinear evolution of drift-tearing modes Phys. Fluids 28 275-7

[13] Dhyani P, Ghosh J, Chattopadhyay P K, Tanna R L, Raju D, Joisa S, Chattopadhyay A K, Basu D, Ramaiya N, Kumar S, Sathyanarayana K, Bhatt S B, Atrey P K, Gupta C N, Rao C V S, Jha R, Saxena Y C and Pal R 2014 A novel approach for mitigating disruptions using biased electrode in Aditya tokamak Nucl. Fusion 54083023

[14] Klüber O, Zohm H, Bruhns H, Gernhardt J, Kallenbach a. and Zehrfeld H P 1991 MHD mode structure and propagation in the ASDEX tokamak Nucl. Fusion $31907-$ 26

[15] Taylor E 2000 Effect of Magnetic Islands on the Local Plasma Behavior in a Tokamak

[16] Fitzpatrick R 1993 Interaction of tearing modes with external structures in cylindrical geometry (plasma) Nucl. Fusion 33 1049-84

[17] Chu M S and Okabayashi M 2010 Stabilization of the external kink and the resistive wall mode Plasma Phys. Control. Fusion 52123001

[18] Fitzpatrick R and Hender T C 1994 Effect of a static external magnetic perturbation on resistive mode stability in tokamaks Phys. Plasmas $13337-55$

[19] Suttrop W, Gruber O, Günter S, Hahn D, Herrmann A, Rott M, Vierle T, Seidel U, Sempf M, Streibl B, Strumberger E, Yadikin D, Neubauer O, Unterberg B, Gaio E, Toigo V and Brunsell P 2009 In-vessel saddle coils for MHD control in ASDEX Upgrade Fusion Eng. Des. 84 290-4

[20] Marrelli L, Bolzonella T, Brunsell P, Cecconello M, Drake J, Franz P, Gregoratto D, Manduchi G, Martin P, Ortolani S, Paccagnella R, Piovesan P, Spizzo G, Yadikin D and Zanca P 2004 MHD control experiments in the Extrap T2R Reversed Field Pinch Am. Phys. Soc. 46th Annu. Meet. Div. Plasma Physics, 15-19 November, 2004, Savannah, GA. Meet. ID DPP04., Abstr. id. NP1.061

[21] Pedrosa M A, García-Cortés I, Brañas B, Balbín R, Hidalgo C, Schmitz L, Tynan G and Post-Zwicker A 1995 On the influence of atomic physics mechanisms on edge 
plasma turbulence in the TJ-I and Princeton Beta Experiment-Modified tokamaks Phys. Plasmas 2 2618-20

[22] Sangwan D, Jha R, Brotankova J and Gopalkrishna M V. 2013 Modification of plasma flows with gas puff in the scrape-off layer of ADITYA tokamak Phys. Plasmas 20

[23] Zweben S J, Stotler D P, Bell R E, Davis W M, Kaye S M, Leblanc B P, Maqueda R J, Meier E T, Munsat T, Ren Y, Sabbagh S A, Sechrest Y, Smith D R and Soukhanovskii V 2014 Effect of a deuterium gas puff on the edge plasma in NSTX Plasma Phys. Control. Fusion Plasma Phys. Control. Fusion 5620

[24] Vershkov V A, Borisov M A, Subbotin G F, Shelukhin D A, Dnestrovskii Y N, Danilov A V, Cherkasov S V, Gorbunov E P, Sergeev D S, Grashin S A, Krylov S V, Kuleshin E O, Myalton T B, Skosyrev Y V and Chistiakov V V 2013 Electron particle transport and turbulence studies in the T-10 tokamak Nucl. Fusion $\mathbf{5 3} 083014$

[25] Carreras B A, Owen L W, Maingi R, Mioduszewski P K, Carlstrom T N and Groebner R J 1998 Effect of edge neutrals on the low-to-high confinement transition threshold in the DIII-D tokamak Phys. Plasmas 5 2623-36

[26] Singh R, Rogister A and Kaw P 2004 The effect of asymmetric gas puffing on toroidal flow in the edge of tokamak plasma Phys. Plasmas 11 129-39

[27] Helander P, Fülöp T and Catto P J 2003 Controlling edge plasma rotation through poloidally localized refueling Phys. Plasmas 10 4396-404

[28] Jha R, Sen A, Kaw P K, Atrey P K, Bhatt S B, Bisai N, Tahiliani K and Tanna R L 2009 Investigation of gas puff induced fluctuation suppression in ADITYA tokamak Plasma Phys. Control. Fusion 51

[29] Sangwan D, Jha R and Tanna R L 2015 Multidirectional plasma flow measurement by Gundestrup Probe in scrape-off layer of ADITYA tokamak Phys. Plasmas 22

[30] Rohollahi A, Elgriw S, Basu D, Wolfe S, Hirose A and Xiao C 2017 Modification of toroidal flow velocity through momentum Injection by compact torus injection into the STOR-M tokamak Nucl. Fusion 57056023

[31] Xiao W W, Diamond P H, Kim W C, Yao L H, Yoon S W, Ding X T, Hahn S H, Kim J, Xu M, Chen C Y, Feng B B, Cheng J, Zhong W L, Shi Z B, Jiang M, Han X Y, Nam 
Y U, Ko W H, Lee S G, Bak J G, Ahn J W, Kim H K, Kim H T, Kim K P, Zou X L, Song S D, Song J I, Yu Y W, Rhee T, Kwon J M, Huang X L, Yu D L, Lee K D, Park S I, Jung M, Zoletnik S, Lampert M, Tynan G R, Bae Y S, Kwak J G, Yan L W, Duan X R, Oh Y K, Dong J Q, Team the K and Team the H-2A 2014 ELM mitigation by supersonic molecular beam injection: KSTAR and HL-2A experiments and theory Nucl. Fusion 54023003

[32] Feng X D, Zhuang G, Yang Z J, Xiao J S, Chen J and Hu X W 2014 Observation of the bifurcation of tearing modes due to supersonic gas injected into the J-TEXT plasmas Phys. Lett. A 378 1147-52

[33] Dudson B 2013 BOUT++ Users Manual

[34] Tanna R L, Ghosh J, Chattopadhyay P K, Raj H, Patel S, Dhyani P, Gupta C N, Jadeja K A, Patel K M, Bhatt S B, Panchal V K, Patel N C, Chavda C, Praveenlal E V, Shah K S, Makawana M N, Jha S K, Gopalkrishana M V, Tahiliani K, Sangwan D, Raju D, Nagora U, Pathak S K, Atrey P K, Purohit S, Raval J, Joisa Y S, Rao C V S, Chowdhuri M B, Banerjee S, Ramaiya N, Manchanda R, Thomas J, Kumar A, Ajay K, Sharma P K, Kulkarni S V, Sathyanarayana K, Shukla B K, Das A, Jha R, Saxena Y C, Sen A, Kaw P K and Bora D 2017 Overview of recent experimental results from the Aditya tokamak Nucl. Fusion 57102008

[35] Patel N, Chavda C, Bhatt S B, Chattopadhyay P and Saxena Y C 2012 Programmable Pulse Generator for Aditya Gas Puffing System J. Phys. Conf. Ser. 390012012

[36] Tanna R, Raj H, Ghosh J, Kumar R, Aich S, Macwan T, Kumawat D, Jadeja K, Patel K M, Kalal M, Varia D, Sadharakiya D, Bhatt S, Sathyanarayana K, Shukla B K, Chattopadhyay P, Makawana M, Shah K S, Gupta S, Ranjan V, Balakrishnan V, Gupta C, Panchal V, Edappala P, Arambhadiya B, Shah M, Raulji V, Chowdhuri M B, Banerjee S, Manchanda R, Shukla G, Shah K, Dey R, Yadava N, Patel S, Bisai N, Raju D, Atrey P K, Pathak S, Nagora U, Raval J, Joisa Y, Kumar M, Tahiliani K, Jha S, Gopalkrishana M and Sen A 2019 Overview of operation and experiments in the ADITYA-U tokamak Nucl. Fusion 59112006

[37] Raj H, Ghosh J, Tanna R L, Chattopadhyay P K, Raju D, Jha S K, Raval J, Joisa Y S, Purohit S, Atrey P K, Saxena Y C and Pal R 2018 Generation and transport of runaway electrons during sawteeth crash in the ADITYA tokamak Nucl. Fusion 58076004 
[38] Raj H 2018 Origin of Harmonics of Drift Tearing Mode in ADITYA tokamak 27th IAEA Fusion Energy Conference - IAEA CN-258

[39] Manchanda R, Ghosh J, Chattopadhyay P K, Chowdhuri M B, Banerjee S, Ramasubramanian N, Patel K M, Kumar V, Vasu P, Tanna R L, Paradkar B, Gupta C N, Bhatt S B, Raju D, Jha R, Atrey P K, Joisa S, Rao C V S and Saxena Y C 2010 Drift-Alfven waves induced optical emission fluctuations in Aditya tokamak Phys. Plasmas 17072515

[40] Jha R, Sen A, Kaw P K, Atrey P K, Bhatt S B, Bisai N, Tahiliani K, Tanna R L and Team A 2009 Investigation of gas puff induced fluctuation suppression in ADITYA tokamak Plasma Phys. Control. Fusion 5117

[41] Spineanu F and Vlad M 2016 A MHD invariant and the confinement regimes in a tokamak Nucl. Fusion 56092005

[42] Grasso D, Ottaviani M and Porcelli F 2001 Linear stability and mode structure of drift tearing modes Phys. Plasmas 8 4306-17

[43] Bisai N, Banerjee S and Sangwan D 2018 Modification of plasma flows in edge and SOL regions by influence of neutral gas Phys. Plasmas 25102503

[44] Dey R, Ghosh J, Chowdhuri M B, Manchanda R, Banerjee S, Ramaiya N, Sharma D, Srinivasan R, Stotler D P and Team A 2017 Investigation of neutral particle dynamics in Aditya tokamak plasma with DEGAS2 code Nucl. Fusion 57086003

[45] Dey R, Chowdhuri M B, Ghosh J, Manchanda R, Yadava N, Ramaiya N, Banerjee S, Nagora U C, Atrey P K, Raval J V, Shankar Joisa Y, Tanna R L, Stotler D P and Team A 2019 Investigation of atomic and molecular processes in $\mathrm{H}_{\alpha}$ emission through modelling of measured $\mathrm{H}_{\alpha}$ emissivity profile using DEGAS2 in the ADITYA tokamak Nucl. Fusion $\mathbf{5 9} 076005$ 